\title{
Trivalent Chromium: A Neglected Latent Contaminant
}

\section{Zhaohui Wang*, Renlan Liu and Jianshe Liu}

State Environmental Protection Engineering Center for Pollution Treatment and Control in Textile Industry, College of Environmental Science and Engineering, Donghua University, Shanghai, 201620, China

As the $21^{\text {st }}$ most abundant element in earth's crust, chromium has been extensively used in industrial activities such as paint pigments and leather tanning. As a consequence, there is a continual influx of chromium contaminants into the environment, thereby posing a serious environmental problem. Chromium exhibits a wide range of possible oxidation states, where trivalent chromium [Cr (III)] is most stable energetically, but $\mathrm{Cr}$ (III) and Cr (VI) are most commonly observed in aquatic environment. $\mathrm{Cr}$ (VI) species are of great environment concern due to their well-known toxicity, carcinogentic properties and high mobility in environmentally relevant media. $\mathrm{Cr}$ (III) is generally regarded as benign and even essential for human and animal, since trace amounts of $\mathrm{Cr}$ (III) is required for sugar and lipid metabolism. In this context, it is a common strategy in environmental remediation to reduce toxic $\mathrm{Cr}$ (VI) to $\mathrm{Cr}$ (III) by various redox reactions. $\mathrm{Cr}$ (III) is thought to be immobilized by precipitating into insoluble $\mathrm{Cr}$ (III) hydroxides [1].

Is it really the case that $\mathrm{Cr}$ (III) is completely safe in this remediation scheme? Recent investigations indicated that the environmental fate of $\mathrm{Cr}$ (III) species is far form being well understood. Cr (III) chemistry is dominated by the formation of stable hexacoordinate complexes with both inorganic and organic ligands. In the absence of organic ligands, hexaaquachromium (III) is the dominant $\mathrm{Cr}$ species under strongly acidic conditions. This inorganic Cr (III) complexes undergo hydrolysis at $\mathrm{pH}>4$, leading to a rapid precipitation of multinuclear $\mathrm{Cr}$ (III) hydroxides at neutral $\mathrm{pH}$. In contrast to its aqua complexes, $\mathrm{Cr}$ (III) is readily organically complexed with carboxylic acids and amino acids so as to make it remain soluble for a long time. Therefore, it is worthy to note that the description of $\mathrm{Cr}$ (III) insolubility at neutral $\mathrm{pH}$ is only correct for solutions without strong organic ligands that out compete $\mathrm{H}_{2} \mathrm{O}$ for $\mathrm{Cr}$ (III) [2]. Formation of stable organo-Cr (III) complexes during $\mathrm{Cr}(\mathrm{VI})$ reduction can increase their environmental mobility and maintain $\mathrm{Cr}$ (III) solubility even at neutral $\mathrm{pH}$.

These soluble Cr (III) complexes with small organic molecules are often neglected and indeed deserve careful investigations on their mobility and particularly their potential transformation to $\mathrm{Cr}$ (VI) in different environmental compartments. However, there is very limited knowledge about environmental oxidation of $\mathrm{Cr}$ (III) to $\mathrm{Cr}$ (VI) [1]. $\mathrm{H}_{2} \mathrm{O}_{2}$ is known to oxidize $\mathrm{Cr}$ (III) at $\mathrm{pH}>7$, but reduces $\mathrm{Cr}$ (VI) at acidic $\mathrm{pH}$. In soils, $\mathrm{Mn}$ (IV) oxides are regarded as the sole oxidant for $\mathrm{Cr}$ (III) transformation, however, it is no longer efficient if insoluble $\mathrm{Cr}$ (III) solid blocks the surface sites of Mn oxides. Currently there is increasing evidence that photo oxidation is an alternative pathway to convert Cr (VI) to Cr (III) [3-6]. Zhang et al. [3] first reported lightinduced oxidation of $\mathrm{Cr}$ (III) by $\cdot \mathrm{OH}$ radicals resulting from photolysis of $\mathrm{Fe}(\mathrm{III})-\mathrm{OH}$ species (especially $\mathrm{Fe}(\mathrm{OH})^{2+}$ ), suggesting its potential for an important oxidation pathway in atmospheric waters. Recently Wang et al. [4] observed that $\mathrm{Cr}$ (III) can be oxidized in Fe (III)-oxalate (Ox)-Cr (III) systems at pH 3.0, however, no Electron Spin Resonance (ESR) signal of $\mathrm{Cr}(\mathrm{V})$-Ox was measured during $\mathrm{Cr}$ (III) oxidation. In contrast, as for $\mathrm{Fe}$ (III)-Ox-Cr (VI) solutions, formation and decay of $\mathrm{Cr}$ (V) species can be detected by ESR upon UV irradiation. This indicates that $\mathrm{Cr}(\mathrm{V})$ is an important intermediate for $\mathrm{Cr}(\mathrm{VI})$ reduction, but not the case for $\mathrm{Cr}$ (III) oxidation in irradiated Fe (III)-Ox-Cr (III) system. Wang et al. [4] proposed that $\mathrm{Cr}(\mathrm{IV})-\mathrm{Ox}$ complexes were formed during $\mathrm{Cr}$ (III) transformation, which were stable at acidic $\mathrm{pH}$ but would be decomposed under alkaline conditions, thus greatly enhancing the environmental risk of $\mathrm{Cr}$ (VI) release. The further investigation by Dai et al. [5] indicated that photo oxidation of $\mathrm{Cr}$ (III)citrate (Cit) without $\mathrm{Fe}$ (III) also can transform $\mathrm{Cr}$ (III) to $\mathrm{Cr}$ (VI). Rates of $\mathrm{Cr}$ (III) oxidation were not sensitive to $\mathrm{pH}$ within the range of 7 to 9 , but increased significantly at higher $\mathrm{pH}$. Cr (II) was firstly generated by a Ligand-to-Metal-Charge-Transfer (LMCT) after excitation of photoactive [Cr (III)-Cit-OH] - species, and then was oxidized to $\mathrm{Cr}$ (VI) via a multiple step pathway. Similar to natural systems, Cr (III) oxidation is also found in water treatment process such as chlorination. Rates of Cr (VI) formation from Cr (III) nitrate, Cr (III)-EDTA and $\mathrm{Cr}$ (III) hydroxide were rapid via chlorine oxidation, on the order of hours. It manifests the possibility of transformation of $\mathrm{Cr}$ (III) to $\mathrm{Cr}$ (VI) during chlorination of drinking water [7].

Therefore, it is urgent and critical to better understand the fate of soluble Cr (III) species and their release risk as latent Cr (VI) precursor. Due to the complicated nature of chromium chemistry, chemical kinetic modeling and quantum chemical calculations may be required as effective tools to predict the kinetics and intermediates of Cr (III) oxidation. Secondly, ESR is a powerful and highly sensitive spectroscopic method to reveal the underlying mechanism for $\mathrm{Cr}$ (III) transformation [8]. However, it should be emphasized here that only organo-Cr (V) or Cr (V)/peroxo, and Cr (III) in some cases are ESRsensitive [4]. It is therefore expected that the advances in fundamental of $\mathrm{Cr}$ (III) chemistry from a viewpoint of chemical kinetics and theoretical calculations, will benefit the in-depth exploration of uncertain fate of $\mathrm{Cr}$ (III) in the environment.

\section{Acknowledgement}

This study was supported by State Key Laboratory of Pollution Control and Resource Reuse Foundation (No. CRRF11023), and the Fundamental Research Funds for the Central Universities (12D11317).

\section{References}

1. Kimbrough DE, Cohen Y, Winer AM, Creelman L, Mabuni C (1999) A Critical Assessment of Chromium in the Environment. Crit Rev Environ Sci Technol 29: $1-46$.

2. Zhitkovich A (2011) Chromium in drinking water: sources, metabolism and cancer risks. Chem Res Toxicol 24: 1617-1629.

3. Zhang H, Bartlett R (1999) Light-Induced Oxidation of Aqueous Chromium (III) in the Presence of Iron (III). Environ Sci Technol 33: 588-594.

4. Wang Z, Ma W, Chen C, Zhao J (2008) Photochemical coupling reactions between $\mathrm{Fe}(\mathrm{III}) / \mathrm{Fe}(\mathrm{II}), \mathrm{Cr}(\mathrm{VI}) / \mathrm{Cr}(\mathrm{III})$ and polycarboxylates: inhibitory effect of $\mathrm{Cr}$ species. Environ Sci Technol 42: 7260-7266.

*Corresponding author: Zhaohui Wang, Associate professor, College of Environmental Science and Engineering, Donghua University, 201620, China, Tel: +86-21-67792557; E-mail: zhaohuiwang@dhu.edu.cn

Received April 28, 2012; Accepted April 28, 2012; Published May 02, 2012

Citation: Wang Z, Liu R, Liu J (2012) Trivalent Chromium: A Neglected Latent Contaminant. Vitam Trace Elem 1:e115. doi:10.4172/2167-0390.1000e115

Copyright: ( 2012 Wang Z, et al. This is an open-access article distributed under the terms of the Creative Commons Attribution License, which permits unrestricted use, distribution, and reproduction in any medium, provided the original author and source are credited. 
Citation: Wang Z, Liu R, Liu J (2012) Trivalent Chromium: A Neglected Latent Contaminant. Vitam Trace Elem 1:e115. doi:10.4172/21670390.1000 e115

5. Dai R, Yu C, Liu J, Lan Y, Deng B (2010) Photo-oxidation of Cr (III)-citrate complexes forms harmful $\mathrm{Cr}(\mathrm{VI})$. Environ Sci Technol 44: 6959-6964.

6. Stasicka Z (2011) Transition Metal Complexes as Solar Photocatalysts in the Environment: A Short Review of Recent Development. In: van Eldik R, Stoche G (Ed) Advances in Inorganic Chemistry. Academic Press, Burlington 63: 291 343
7. Lindsay DR, Farley KJ, Carbonaro RF (2012) Oxidation of $\mathrm{Cr}$ (III) to $\mathrm{Cr}$ (VI) during chlorination of drinking water. J Environ Monit.

8. Wang $\mathrm{ZH}, \mathrm{Ma}$ WH, Chen CC, Ji HW, Zhao JC (2011) Probing paramagnetic species in titania-based heterogeneous photocatalysis by electron spin resonance (ESR) spectroscopy - A mini review. Chem Eng J 170: 353-362. 